\title{
Succinate dehydrogenase inhibition leads to epithelial-mesenchymal transition and reprogrammed carbon metabolism
}

Paul-Joseph P Aspuria" ${ }^{*}$, Sophia Y Lunt ${ }^{2,3 \dagger}$, Leif Väremo ${ }^{4 \dagger}$, Laurent Vergnes ${ }^{5}$, Maricel Gozo ${ }^{1,6}$, Jessica A Beach ${ }^{1,6}$, Brenda Salumbides ${ }^{1}$, Karen Reue ${ }^{5}$, W Ruprecht Wiedemeyer ${ }^{1,7}$, Jens Nielsen ${ }^{4}$, Beth Y Karlan ${ }^{1,7}$ and Sandra Orsulic ${ }^{1,7}$

\begin{abstract}
Background: Succinate dehydrogenase (SDH) is a mitochondrial metabolic enzyme complex involved in both the electron transport chain and the citric acid cycle. SDH mutations resulting in enzymatic dysfunction have been found to be a predisposing factor in various hereditary cancers. Therefore, SDH has been implicated as a tumor suppressor.

Results: We identified that dysregulation of SDH components also occurs in serous ovarian cancer, particularly the SDH subunit SDHB. Targeted knockdown of Sdhb in mouse ovarian cancer cells resulted in enhanced proliferation and an epithelial-to-mesenchymal transition (EMT). Bioinformatics analysis revealed that decreased SDHB expression leads to a transcriptional upregulation of genes involved in metabolic networks affecting histone methylation. We confirmed that $S d h b$ knockdown leads to a hypermethylated epigenome that is sufficient to promote EMT. Metabolically, the loss of $S \mathrm{dhb}$ resulted in reprogrammed carbon source utilization and mitochondrial dysfunction. This altered metabolic state of $S d h b$ knockdown cells rendered them hypersensitive to energy stress.

Conclusions: These data illustrate how SDH dysfunction alters the epigenetic and metabolic landscape in ovarian cancer. By analyzing the involvement of this enzyme in transcriptional and metabolic networks, we find a metabolic Achilles' heel that can be exploited therapeutically. Analyses of this type provide an understanding how specific perturbations in cancer metabolism may lead to novel anticancer strategies.
\end{abstract}

Keywords: Succinate dehydrogenase, SDH, Ovarian cancer, EMT, Carbon metabolism, Epigenetics

\section{Background}

Ovarian cancer is the most lethal gynecological malignancy in the United States, resulting in approximately 22,000 new diagnoses and 14,000 deaths in 2013 [1]. The prognosis of ovarian cancer is poor and has seen limited improvement in the past few decades with a 5-year survival rate of roughly 44\% [2]. High-grade serous ovarian cancer is the most common and most lethal subtype of ovarian cancer, contributing to $>70 \%$ of deaths from ovarian cancer [3]. Integrative large-scale analyses of patient tumors with serous histology have revealed that the disease is highly heterogeneous but can be clustered into

\footnotetext{
* Correspondence: Paul-Joseph.Aspuria@cshs.org

${ }^{\dagger}$ Equal contributors

'Women's Cancer Program, Samuel Oschin Comprehensive Cancer Institute, Cedars-Sinai Medical Center, 8700 Beverly Blvd., Los Angeles, CA 90048, USA Full list of author information is available at the end of the article
}

several molecular subtypes with different clinical outcomes $[4,5]$. One of the poor prognosis subtypes contains an epithelial-mesenchymal transition (EMT) signature [5]. Therefore, understanding and characterizing EMT in ovarian cancer is of great importance.

EMT is a biological process that converts epithelial cells to motile, mesenchymal cells [6]. It is largely defined by the loss of plasma membrane E-cadherin and conversion from a cobblestone-like morphology to a more spindleshaped and less adherent state. It was initially characterized as a fundamental event in morphogenesis during embryonic tissue and organ development [7]. However, emerging evidence suggests that cancer cells utilize the EMT program to enhance invasiveness, increase migratory ability, and acquire stem-like characteristics such as chemoresistance. EMT reprogramming involves several layers of

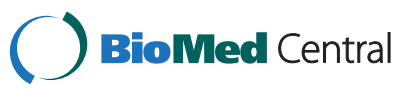


regulation and has been the subject of intense research, especially in the context of cancer [6]. One area that has not been extensively studied is the role of metabolic enzymes in the regulation and maintenance of EMT.

Alterations in metabolism have been implicated in cancer, with the main focus on the Warburg effect, a phenomenon in which cancer cells upregulate glycolysis and lactate production while decreasing glucose contribution to the citric acid (TCA) cycle in the mitochondria, even in the presence of sufficient oxygen [8-10]. However, mitochondrial metabolism is also important for cancer proliferation. For example, mutations in the mitochondrial enzyme succinate dehydrogenase $(\mathrm{SDH})$ have been reported in a wide variety of cancers [11,12]. Also known as mitochondrial respiratory Complex II, SDH is the only enzyme to participate in both the TCA cycle and electron transport chain (ETC.). In the TCA cycle, SDH catalyzes the oxidation reaction where succinate is converted to fumarate, which is coupled to the reduction of ubiquinone to ubiquinol in the ETC. [12]. SDH is a holoenzyme consisting of four essential subunits: SDHA (a flavoprotein), SDHB (iron-sulfur protein), and two membrane anchor units SDHC and SDHD. SDH assembly requires two factors, SDHAF1 and SDHAF2 [13], while its function is influenced by the deacetylase activity of SIRT3 [14]. SDHx loss-of-function mutations have been found to predispose individuals to hereditary pheochromocytoma (PCC), paraganglioma (PGL), gastrointestinal stromal tumor (GIST), and renal cell carcinoma [12]. Interestingly, it has been shown that $S D H x$ mutations are associated with hallmarks of EMT in PCC and PGL as a result of alterations to the epigenome $[15,16]$. Furthermore, the SDH regulators SDHAF1 and SIRT3 have been identified as tumor suppressor genes in lung and breast cancer, respectively $[14,17]$. This has led to the definition of SDH as a mitochondrial tumor suppressor and a key player in cancer cell differentiation.

Ovarian cancer is characterized by a remarkable degree of genomic disarray but extremely low frequency of recurrent genomic alterations [4]. The dearth of common therapeutic targets in ovarian cancer samples highlights the need for individualized therapy or different approaches to identifying patient groups with common characteristics that can be therapeutically exploited. Interestingly, succinate was identified as a potential metabolic urinary biomarker for ovarian cancer [18], indicating that altered SDH activity may be important in ovarian cancer progression.

Here, we investigate whether alterations in SDH function also impact ovarian cancer biology. We find that dysregulation of SDH members occurs frequently in serous ovarian cancer. Utilizing a mouse ovarian cancer cell line as a model system, we find that knockdown of one of the $\mathrm{SDH}$ subunits, $S d h b$, increases aggressiveness and results in EMT. Sdhb knockdown cells were found to have a hypermethylated epigenome whereby increased H3K27 methylation was sufficient to induce EMT. Using a metabolomic approach to investigate the metabolic effects of decreased SDH activity, we found that $S d h b$ knockdown leads to altered glucose and glutamine utilization through central carbon metabolism as well as mitochondrial dysfunction. These data demonstrate the metabolic and epigenetic consequences of decreased activity of a mitochondrial metabolic enzyme in ovarian cancer.

\section{Methods}

\section{Plasmids and reagents}

pLKO.1-based short hairpin constructs specific for mouse $S d h b$ and human $S D H B$, as well as scrambled control sequences, were obtained from Sigma-Aldrich (St. Louis, MO, USA). shRNA sequences are listed in Additional file 1: Table S1. GSK-J1 and GSK-J4 were obtained from Tocris Bioscience (Bristol, Avon, UK). Metformin, p-iodonitrotetrazolium, oligomycin, carbonyl cyanide 4(trifluoromethoxy)phenylhydrazone chloride (FCCP), and rotenone-myxothiazol were purchased from SigmaAldrich.

\section{Cell lines}

C1 mouse ovarian cancer cells were generated as previously described [19-21]. HEY cells were obtained from American Type Culture Collection (ATCC). Both cell lines were grown in Dulbecco's modified Eagle's medium (DMEM) supplemented with 10\% FBS unless otherwise indicated.

\section{Lentiviral-mediated gene knockdown}

pLKO.1-sh1-Sdhb, pLKO.1-sh2-Sdhb, pLKO.1-sh1-SDHB, pLKO.1-sh2-SDHB, and pLKO.1 scrambled shRNA plasmids were co-transfected with the delta 8.9 packaging plasmid and VSVG plasmid into Lenti-X ${ }^{\mathrm{TM}} 293 \mathrm{~T}$ cells using Lipofectamine 2000 (Invitrogen, Carlsbad, CA, USA). After overnight incubation, the cell culture medium was changed to DMEM supplemented with $30 \%$ FBS. The medium was harvested $48 \mathrm{~h}$ later and filtered through a $0.45-\mu \mathrm{M}$ filter. The viral supernatant was used for infection of $\mathrm{C} 1$ and HEY cells with polybrene $(4 \mu \mathrm{g} / \mathrm{ml})$. Cells were infected overnight, and polyclonal populations of stable transductants were selected with puromycin $(5 \mu \mathrm{g} / \mathrm{ml})$ for $72 \mathrm{~h}$.

\section{Western blot analysis}

Cell lysates were prepared using RIPA lysis buffer (Sigma-Aldrich) containing a protease inhibitor cocktail (Roche, Basel, Switzerland). Protein concentrations were quantified by the BCA protein assay (Thermo Scientific, Rockford, IL, USA). Fifty micrograms of lysate was loaded onto a gradient gel (BioRad, Hercules, CA, USA) and 
subjected to gel electrophoresis. Semi-dry protein transfer to a PVDF nitrocellulose membrane (BioRad) was performed using the Transblot Turbo transfer system (BioRad). The membrane was then blocked in Odyssey blocking buffer (LiCOR, Lincoln, NE, USA) for $30 \mathrm{~min}$ and incubated with the appropriate antibody overnight at $4^{\circ} \mathrm{C}$. Antibodies included E-cadherin (BD Biosciences, San Jose, CA, USA), $\beta$-actin (Abcam, Cambridge, MA, USA), Complex II Western Blot Antibody Cocktail that detects SDHB (30 kDa) and Complex Va $(60 \mathrm{kDa})$ (Abcam, Burlungame, CA, USA), Methyl-Histone H3 Antibody Sampler Kit (Cell Signaling, Danvers, MA, USA), and SDHB (Santa Cruz Biotechnology, Dallas, TX, USA). Membranes were then washed with TBS-T, incubated with the appropriate secondary antibody for $1 \mathrm{~h}$ at room temperature and then washed with TBS-T. The membrane signal was subsequently analyzed by the LiCOR Odyssey system (LiCOR Biosciences, Frankfurt, Germany).

\section{qRT-PCR analysis}

RNA was extracted using the RNeasy Miniprep Kit (Qiagen, Valencia, CA, USA) and was reverse transcribed to cDNA using the Quantitect Reverse Transcription Kit (Qiagen). For qRT-PCR, 50 ng of cDNA was mixed with the appropriate primers and the iQ SYBR-Green Supermix (BioRad) in a 96-well plate format. The qRT-PCR reaction was performed using the CFX96 Real-Time System (BioRad). Primer sequences are listed in Additional file 1: Table S1.

\section{Database mining}

OncoPrints and the mRNA vs. CAN plot were generated using the cBio Cancer Genomics Portal (http://www. cbioportal.org) [22]. Tumorscape was analyzed as described [23].

\section{Immunofluorescence microscopy}

Cells grown on coverslips were fixed with 3\% paraformaldehyde for $20 \mathrm{~min}$, washed with PBS, then permeabilized with $0.1 \%$ Triton $\mathrm{X}-100 / \mathrm{PBS}$ for $10 \mathrm{~min}$. This was followed by incubation for $1 \mathrm{~h}$ at $37^{\circ} \mathrm{C}$ with E-cadherin antibody (1:1,000 dilution; BD Biosciences) or SDHB antibody (1:100 dilution; Santa Cruz Biotechnology). After washing with PBS, cells were incubated with appropriate fluorescent secondary antibody (1:1,000 dilution; Invitrogen) for $30 \mathrm{~min}$ at $37^{\circ} \mathrm{C}$. The coverslips were mounted on slides with Vectashield mounting medium with DAPI (Vector Labs, Burlingame, CA, USA).

\section{Succinate dehydrogenase assay}

The SDH assay was performed using the Succinate Dehydrogenase Activity Colorimetric Assay Kit (Biovision, Milpitas, California, USA). Briefly, $1 \times 10^{7}$ cells were dounce homogenized in $1 \mathrm{ml} \mathrm{SDH}$ assay buffer. Five microliters of cell lysate were added to a mixture containing SDH assay buffer, SDH substrate mix, and SDH probe. Absorbance readings at $600 \mathrm{~nm}$ were taken every $3 \mathrm{~min}$ for a total of $30 \mathrm{~min}$. SDH activity was determined by the formula $B /(\Delta T \times V) \times$ Dilution factor, where $B=$ amount of reduced DCIP from standard curve ( $\mathrm{nmol}), \Delta T=$ reaction time ( $\mathrm{min}), V=$ sample volume added into the reaction well $(\mu \mathrm{l})$, and $D=$ dilution factor.

\section{Cell proliferation and drug treatment}

Cells were seeded onto 6 -well or 96 -well plates at $5 \times 10^{4}$ or $1 \times 10^{3}$ cells/well, respectively. For the glucose and glutamine withdrawal experiments, the medium was changed to DMEM $+10 \%$ FBS with indicated concentrations of glucose or glutamine $24 \mathrm{~h}$ post-seeding. To assay cell proliferation, triplicate cultures were trypsinized at the indicated time points and counted using the trypan blue exclusion method. To assay metformin sensitivity, various concentrations of metformin were added to cultures in a 6 -well plate $24 \mathrm{~h}$ post-seeding and cells were stained with crystal violet at the indicated time points.

\section{Soft agar growth}

To assay anchorage-independent growth, $1 \times 10^{4}$ cells were mixed with $0.5 \%$ agarose and overlayed on a $0.9 \%$ agarose base layer in a 6-well plate. Plates were incubated at $37^{\circ} \mathrm{C}$ for 3 weeks in a $5 \% \mathrm{CO}_{2}$ incubator. Colonies were stained with p-iodonitrotetrazolium chloride overnight and counted using a stereomicroscope. Colony size was measured using QCapture Pro ${ }^{\mathrm{rм}}$ (Q Imaging, Surrey, BC, Canada).

\section{Isotope labeling studies}

For carbon tracing through central carbon metabolism, cells were cultured for approximately $24 \mathrm{~h}$ in $10-\mathrm{cm}$ plates in glucose- and glutamine-free DMEM (Sigma D5030, Sigma-Aldrich) containing 10\% dialyzed FBS, $1 \%$ streptomycin/penicillin, naturally labeled $4 \mathrm{mM}$ glutamine or $25 \mathrm{mM}$ glucose, and the appropriate tracer, [U- ${ }^{13} \mathrm{C}_{6}$ ] glucose or $\left[\mathrm{U}_{-}{ }^{13} \mathrm{C}_{5}\right.$ ] glutamine (Cambridge Isotopes Laboratories, Inc., Cambridge, MA, USA). After incubation, the medium was aspirated and each plate was rinsed with $10 \mathrm{ml}$ ice-cold saline. The saline was aspirated, and cells were quenched with $2.89 \mathrm{ml}$ of $-20^{\circ} \mathrm{C}$ HPLC-grade methanol. After adding $1.74 \mathrm{ml}$ of ice water, the cells were scraped with a cell lifter and collected in 15-ml conical tubes. Chloroform $\left(2.89 \mathrm{ml}\right.$ at $\left.-20^{\circ} \mathrm{C}\right)$ was added to each tube and vortexed for $10 \mathrm{~min}$ at $4^{\circ} \mathrm{C}$. Extracts were centrifuged at $4,000 \times g$ for $15 \mathrm{~min}$ at $4^{\circ} \mathrm{C}$. The upper aqueous phase was collected in a separate tube and evaporated under nitrogen for polar metabolite analysis. The metabolites were analyzed using liquid chromatography tandem mass spectrometry (LC-MS/MS) using a variation of the method described previously [24]. A Paradigm MS4 HPLC 
(Michrom Bioresources, Auburn, CA, USA) and a Synergi Hydro column ( $4 \mu \mathrm{m}$ particle size, $80 \AA$, $150 \mathrm{~mm} \times 2 \mathrm{~mm}$, from Phenomenex, Torrance, CA, USA) were used for the separation of metabolites by polarity. Prior to column separation, the samples were loaded onto a trapping column (C18, $4 \mathrm{~mm} \times 2 \mathrm{~mm}$, from Phenomenex) and washed for $30 \mathrm{~s}$ with HPLC grade water containing $10 \mathrm{mM}$ tributylamine and $15 \mathrm{mM}$ acetic acid for rapid desalting. HPLC separation was coupled with negative-mode electrospray ionization (ESI) to a TSQ Vantage triple stage quadrupole mass spectrometer (Thermo Scientific, Waltham, MA, USA) operating in multiple reactions monitoring (MRM) mode. The LC parameters were as follows: autosampler temperature, $10^{\circ} \mathrm{C}$; injection volume, $10 \mu \mathrm{l}$; column temperature, room temperature; and flow rate, $200 \mu \mathrm{l} /$ minute. The LC solvents were Solvent A: $10 \mathrm{mM}$ tributylamine and $15 \mathrm{mM}$ acetic acid in 97:3 water:methanol ( $\mathrm{pH} 4.95)$, and Solvent B: methanol. Elution from the column was performed over 50 minutes with the following gradient: $t=0,0 \% \mathrm{~B} ; t=5,0 \% \mathrm{~B} ; t=10,20 \% \mathrm{~B} ; t=20,20 \% \mathrm{~B} ; t=35$, $65 \% \mathrm{~B} ; t=38,95 \% \mathrm{~B} ; t=42,95 \% \mathrm{~B}, t=43,0 \% \mathrm{~B} ; t=50,0 \%$ B. ESI spray voltage was $3,000 \mathrm{~V}$. Nitrogen was used as sheath gas at 30 psi and as the auxiliary gas at $10 \mathrm{psi}$, and argon as the collision gas at 1.5 mTorr, with the capillary temperature at $325^{\circ} \mathrm{C}$. Scan time for each MRM transition was 0.1 second with a scan width of $1 \mathrm{~m} / \mathrm{z}$. The $\mathrm{LC}$ runs were divided into time segments, with the MRM scans within each time segment containing compounds eluting during that time interval. For compounds eluting near boundaries between time segments, the MRM scan corresponding to the compound was conducted in both time segments. Instrument control, chromatographic control, and data acquisition were performed by the Xcalibar software (Thermo Scientific). Data analysis was performed using MAVEN [25,26]. The protein content of extracted cells was determined from the dried protein fraction from each extraction, which was incubated overnight in $2 \mathrm{ml}$ of $0.2 \mathrm{M} \mathrm{KOH}$ and quantified by Bradford assay. Isotope labeling data was corrected for the natural abundance of different isotopes using IsoCor [27].

\section{Glucose consumption and lactate excretion rates}

Glucose and lactate levels in the media were analyzed at time 0 and after $72 \mathrm{~h}$ using LC-MS/MS. An H-Class UPLC system and AQCUITY UPLC BEH Amide column $(2.1 \times 100 \mathrm{~mm}, 1.7 \mu \mathrm{m}$ particle size, from Waters $)$ were used for the separation of glucose and lactate. UPLC separation was coupled with negative-mode ESI to a Waters Xevo TQ-S mass spectrometer operating in MRM mode. The LC parameters were as follows: autosampler temperature, $5^{\circ} \mathrm{C}$; injection volume, $5 \mu \mathrm{l}$; column temperature, $50^{\circ} \mathrm{C}$; and flow rate, $400 \mu \mathrm{l} / \mathrm{min}$. The LC solvents were solvent A, $50 \mathrm{mM}$ ammonium formate in water $(\mathrm{pH} 3)$ and solvent $\mathrm{B}$, acetonitrile. Elution from the column was performed over 2 min with an isocratic gradient of $40 \%$ solvent $\mathrm{A}$ and $60 \%$ solvent $\mathrm{B}$. The capillary voltage was $2.92 \mathrm{kV}$, and the cone voltage was $50 \mathrm{~V}$. The flow rates of cone gas and desolvation gas were 150 and $600 \mathrm{~L} / \mathrm{h}$, respectively. The source temperature was $150^{\circ} \mathrm{C}$, and the desolvation temperature was $500^{\circ} \mathrm{C}$. Argon was used as collision gas at 1.5 mTorr. Collision energies and source cone potentials were optimized for each transition using Waters QuanOptimize software. Data were acquired using MassLynx 4.1 and QuanLynx software. The following equation was used to calculate metabolite consumption/ excretion per $10^{6}$ cells per hour, denoted as $\alpha$ :

$$
\alpha=\frac{[\text { metabolite }]_{\text {initial }}-[\text { metabolite }]_{\text {final }}}{\frac{X_{\text {initial }}}{\mu} \cdot\left(e^{\mu \Delta t}-1\right)}
$$

where

$$
\mu=\text { specific growth rate }=\frac{1}{t} \cdot \ln \left(\frac{X_{\text {final }}}{X_{\text {initial }}}\right)
$$

and $X$ is the cell number and $t$ is the time in hours.

\section{Cellular oxygen consumption and extracellular acidification rates}

Cellular metabolic rates were measured using an XF24 Analyzer (Seahorse Bioscience, North Billerica, MA, USA) as previously described [28]. Mixing, waiting, and measurement times were 4, 2, and $2 \mathrm{~min}$ and 2, 3, and $3 \mathrm{~min}$ for $\mathrm{C} 1$ and HEY cells, respectively. The measurements were normalized against protein concentration. The concentrations for compounds injected during the analysis were $100 \mu \mathrm{M}$ 2,4 dinitrophenol, $0.5 \mu \mathrm{M}$ oligomycin, $1 \mu \mathrm{M}$ FCCP, $1 \mu \mathrm{M}$ rotenone-myxothiazol and $0.75 \mu \mathrm{M}$ oligomycin, $0.5 \mu \mathrm{M}$ FCCP, $0.75 \mu \mathrm{M}$ rotenone-myxothiazol for $\mathrm{C} 1$ cells and HEY cells, respectively. Data was obtained using the XF24 Analyzer software.

\section{RNA-seq}

Extracted RNA was enriched using the Ribominus ${ }^{\mathrm{TM}}$ Eukaryote Kit (Invitrogen). The Ion $\mathrm{PI}^{\mathrm{Im}}$ Template OT2 200 Kit (Invitrogen) was used to prepare the RNA library for sequencing. Sequencing was performed using the Ion Proton $^{\mathrm{TM}}$ Sequencer (Life Technologies, Grand Island, NY, USA). Fragments per kilobase of exon per million reads (FPKM) were calculated using TopHAT and Cufflinks [29].

\section{Gene set analysis}

Genes with FPKM smaller than 5 were discarded, leaving a total of 6,503 genes for further analysis. The log2 fold change (i.e., $\log 2$ of the FPKM ratio) was used as the gene-level statistic. The gene set analysis was carried out using the R/Bioconductor package piano [30]. Three types of gene set collections were used, describing the 
association between (i) genes and metabolites, (ii) genes and metabolic pathways, and (iii) Gene Ontology (GO) terms.

The human genome-scale metabolic network HMR2 was used to define the metabolite gene sets [31]. HMR2 contains gene associations for its enzymatic reactions, so for each metabolite, all genes that are associated with reactions in which the metabolite takes part are grouped into a gene set. The metabolic pathway gene sets were defined by all genes associated to reactions belonging to an individual pathway. Each set of human genes was translated into a set of mouse genes by mapping the human Ensembl gene identifiers to mouse Ensembl gene identifiers using the $\mathrm{R} /$ Bioconductor package biomaRt [32]. This package was also used to acquire the GO term gene. Gene set analysis was carried out separately for the metabolites, pathways, and GO terms. In all cases, the gene set statistics were calculated using piano with the median method and the gene set $p$ values were calculated using gene permutation (10,000 permutations). The piano package calculates scores for several directionality classes. We primarily relied on the distinct-directional class for the identification of gene sets that are coordinately regulated in a distinct direction (up or down). Supplemental materials and methods are found in Additional file 2.

\section{Results}

\section{SDH pathway members are downregulated in serous ovarian cancer}

The Cancer Genome Atlas (TCGA) provides an exhaustive catalog of molecular changes for specific cancer types [4]. An analysis of the TCGA ovarian cancer dataset revealed significant genomic deletions of SDH members in $67 \%$ of serous ovarian cancer patient samples, with heterozygous deletion of $S D H B$ found in $39 \%$ of the samples (Figure 1A). Heterozygous loss of $S D H B$ correlated with decreased $S D H B$ mRNA levels relative to diploid samples (Figure 1B). Statistically significant focal deletion of $S D H B(q<.01)$ was confirmed in Tumorscape, a dataset that includes whole genome analyses of somatic copy number alterations (CNA) in 110 ovarian cancer samples [23] (Figure 1C). Recurrent hemizygous deletions have been found to enrich for genes involved in growth inhibition thereby optimizing proliferative potential $[33,34]$. It has been proposed that the majority of tumor suppressor genes are likely to be haploinsufficient and selected for during the evolution of a cancer [34]. Homozygous focal deletions are rare in ovarian cancer [4], suggesting that haploinsufficiency may be the preferred mechanism for tumor progression. Immunohistochemical staining of ovarian carcinoma tissue microarray was used to determine levels of SDHB protein in malignant tumor cells relative to adjacent nonmalignant stroma. SDHB protein was detected in cytoplasmic granules in most cells, which is consistent with its predicted localization at the mitochondrial membrane. Of 211 ovarian cancer samples that had sufficient stromal and epithelial components for evaluation, seven samples exhibited significantly decreased levels of SDHB in tumor epithelia relative to tumor stroma (Additional file 3: Figure S1). Together, these data suggest that decreased SDH activity through altered expression of $S D H B$ may be biologically relevant in a subset of ovarian cancers.

\section{Knockdown of Sdhb results in EMT in mouse ovarian cancer cells}

Given our analyses of the TCGA and Tumorscape datasets, we focused on the most frequently downregulated $\mathrm{SDH}$ subunit, $S d h b$. We utilized C1 mouse ovarian cancer cells that are $p 53$ deficient and express $C$-myc and $K$-ras ${ }^{G 12 D}$. These genetically defined cells have been previously used as a system to elucidate the function of putative oncogenes in ovarian cancer [19-21]. Two shRNA constructs targeting $S d h b$ were used to generate stable $S d h b$ knockdown cell lines. Western blotting showed that both constructs (sh1-Sdhb and sh2-Sdhb) decreased the amount of SDHB expression relative to the scrambled control (Figure 2A). This was further confirmed by immunofluorescence (Additional file 4: Figure S2). Sdhb knockdown cell lines (C1-sh1-Sdhb and C1-sh2-Sdhb) showed a significant decrease in SDH activity relative to the control (Figure 2B). Knockdown of $S d h b$ resulted in an increased proliferation rate and an enhanced ability to form colonies in soft agar relative to the control $\mathrm{C} 1$-scr cells (Figure 2C,D). This effect on soft agar growth was also seen upon $S D H B$ knockdown in the human ovarian cancer cell line, HEY (Additional file 5: Figure S3A,B). In addition to their augmented growth capability, $S d h b$ knockdown cells were more elongated and spindle-shaped compared to the cobblestone morphology of the control cells (Figure 2E). This morphological change resembled EMT. To determine the involvement of EMT at the molecular level, we analyzed $S d h b$ knockdown and control cells for membrane localization of E-cadherin, which is frequently lost during EMT. Immunofluorescence analysis revealed E-cadherin staining at the plasma membrane in C1-scr cells (Figure 2E). In contrast, E-cadherin localized within cytoplasmic punctate structures in $S d h b$ knockdown cells (Figure 2E). Quantitative RT-PCR analysis of mRNA levels of various transcription factors involved in EMT (Snai1, Snai2, Twist1, and Twist2) revealed their upregulation in $S d h b$ knockdown cells relative to the control cells, with the most dramatic increase in Twist2 (Figure 2F). Decreased expression of E-cadherin and increased expression of TWIST1/2 in $S d h b$ knockdown cells were also seen on the protein level (Figure 2A). Typically under induction of EMT, cells continue to proliferate at the same level or arrest to promote invasion and migration [35]. However, the increased proliferation in C1 


\section{A}

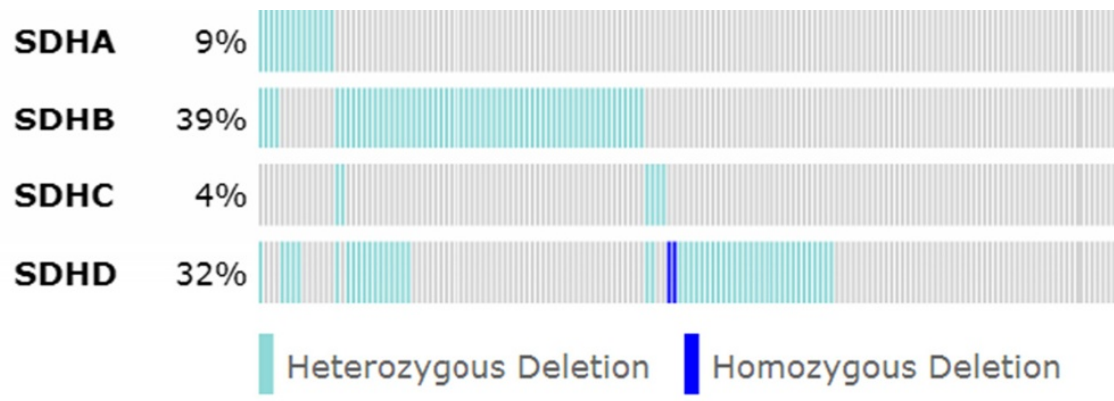

B

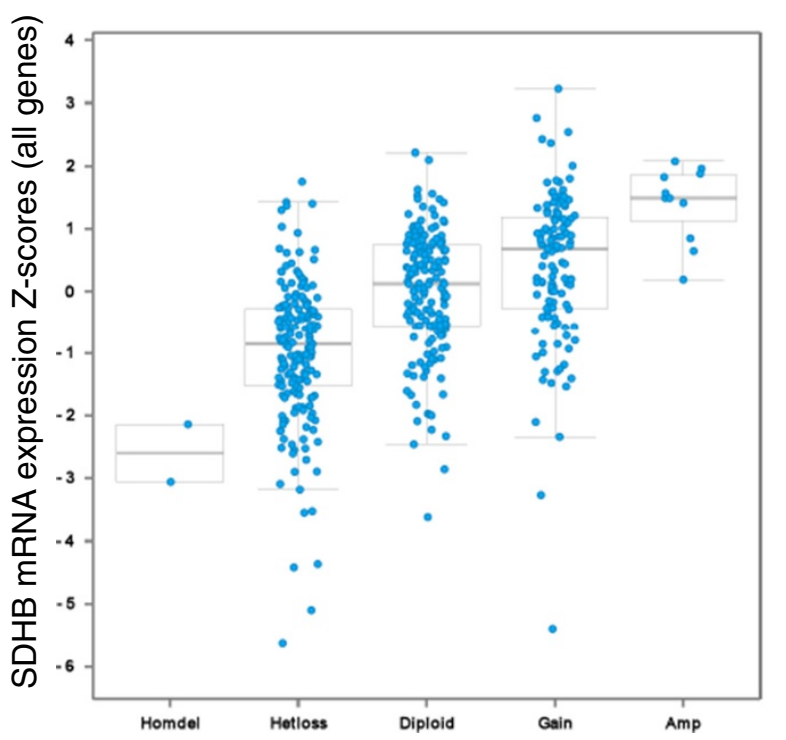

C

\begin{tabular}{cccc}
\hline Gene & Percent with Deletion & q-value \\
\hline & Overall & Focal & \\
SDHA & 8.74 & 0.97 & 1 \\
SDHB & $\mathbf{3 0 . 1}$ & $\mathbf{1 9 . 4 2}$ & $\mathbf{0 . 0 0 6 6 9}$ \\
SDHC & 6.8 & 2.91 & 1 \\
SDHD & 28.16 & 4.85 & 1 \\
\hline
\end{tabular}

SDHB, Putative copy number alterations from GISTIC

Figure 1 SDH components are downregulated in serous ovarian cancer. (A) Oncoprint depicting 575 patient samples with genetic loss of SDH subunits. Light blue and dark blue indicate heterozygous deletion and homozygous deletion, respectively. (B) A plot showing the relationship between SDHB mRNA abundance and copy number alteration of the SDHB gene. (C) Tumorscape copy number data for SDH subunits tabulated. Bold face indicates significance ( $q$ value $<.01)$.

mouse ovarian cancer cells upon $S d h b$ knockdown can be explained by the overriding proliferative signal in these cells due to $c$-myc overexpression. Indeed, several studies have shown that various EMT-inducing factors, especially TWIST1/2, synergize with other oncogenic aberrations to enhance proliferation [36-38]. These data suggest that Sdhb knockdown transforms mouse ovarian cancer cells to a more aggressive and mesenchymal state.

\section{Knockdown of Sdhb leads to histone hypermethylation}

To identify how the loss of Sdhb led to EMT, we investigated the transcriptional regulation of cellular metabolism upon Sdhb knockdown. C1-scr and C1-sh1-Sdhb cells were first subjected to RNA-seq. The extracted data was then integrated with a recently published comprehensive human genome-scale metabolic network, HMR2 [39]. HMR2 represents a holistic view of metabolism by providing associations between metabolites, reactions, and their corresponding enzyme-coding genes, which we mapped to mouse homologs to match the RNA-seq gene annotation. For each metabolite, we constructed a gene set consisting of all enzymes catalyzing reactions in which the metabolite takes part, i.e., genes that control the flux through that metabolite. Next, we performed gene set analysis on the metabolite gene sets using the $\log 2$ fold changes of the genes to score each metabolite [30]. Gene sets (metabolites) found significant in this analysis are termed reporter metabolites [40] and represent hotspots in the metabolic network around which significant transcriptional regulation occurs (Figure 3A, Additional file 6: Table S2). Interestingly, S-adenosyl methionine (SAM), S-adenosyl homocysteine (SAH), and tetrahydrofolate (THF), relevant metabolites in the SAM cycle, were all identified as reporter metabolites $(p<0.005)$ influenced 


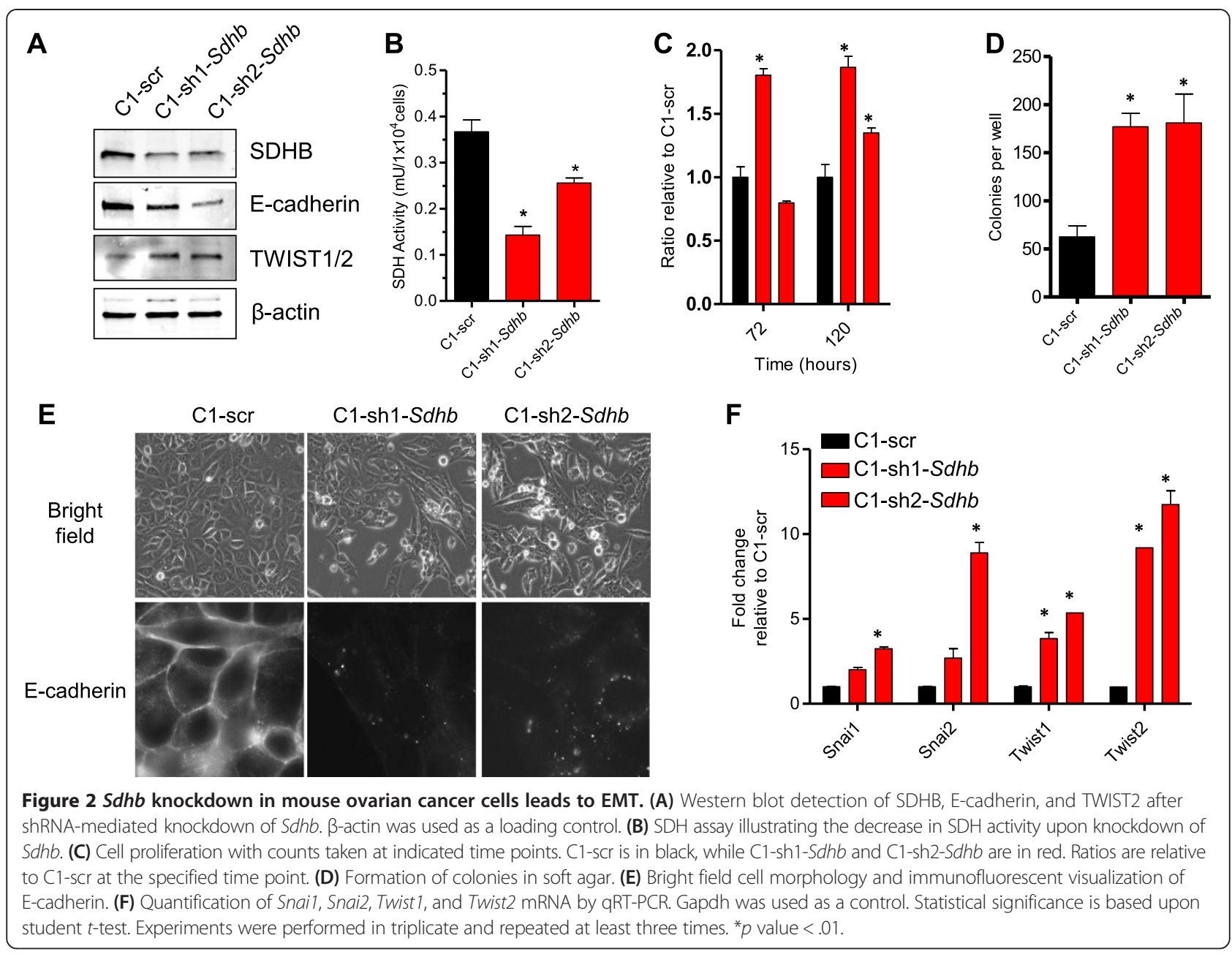

by transcriptional upregulation (Figure 3B). In the SAM cycle, the reactive methyl group in SAM is transferred to a substrate in a transmethylation reaction producing $\mathrm{SAH}$. If the substrate is a histone methyltransferase (HMT), this can subsequently methylate the histones, thus influencing the epigenome.

To further validate the transcriptional influence on histone methylation, we performed an additional gene set analysis using GO terms as gene sets. In Figure 3C, GO terms significantly enriched with upregulated genes $(p<0.05)$ and relevant to histone methylation are shown, clearly indicating a pattern of increased histone methylation regulated on the transcriptional level (complete results are presented in Additional file 6: Table S2). In summary, Sdhb knockdown appears to be orchestrating a transcriptional program in which histone methylation is promoted or maintained, possibly through upregulation of the SAM cycle.

To confirm whether histone methylation was affected, we analyzed by Western blot various methylated histone marks in Sdhb knockdown and control cells. Indeed, $S d h b$ knockdown cells had elevated levels of histone H3 dimethylated $\mathrm{K} 4, \mathrm{~K} 9, \mathrm{~K} 27$, and $\mathrm{K} 36$ marks in comparison to control cells (Figure 4A). We further investigated the aberration in H3K27 methylation because of its known association with malignant progression [41]. There are two known H3K27 demethylases, JMJD3 and UTX, in mammalian cells. Highly selective H3K27 demethylase inhibitors, GSK-J1 and GSK-J4 (a more cell permeable derivative of GSK-J1), have been developed [42]. To determine whether chemical inhibition of H3K27 histone demethylases could lead to EMT, C1-scr cells were treated with DMSO or each inhibitor for 5 days. DMSOtreated cells exhibited cobblestone morphology, while treatment with GSK-J1 or GSK-J4 resulted in a spindleshaped and elongated cell morphology analogous to $S d h b$ knockdown (Figure 4B). To confirm that EMT occurred in cells treated with H3K27 demethylase inhibitors, E-cadherin localization was analyzed by immunofluorescence. Indeed, E-cadherin was localized to punctate cytoplasmic structures in cells treated with GSK-J1 or GSK-J4 as opposed to the plasma membrane staining found in the DMSO-treated control cells (Figure 4C). Cells treated with GSK-J1 and GSK-J4 also had elevated levels of Snai2 and 


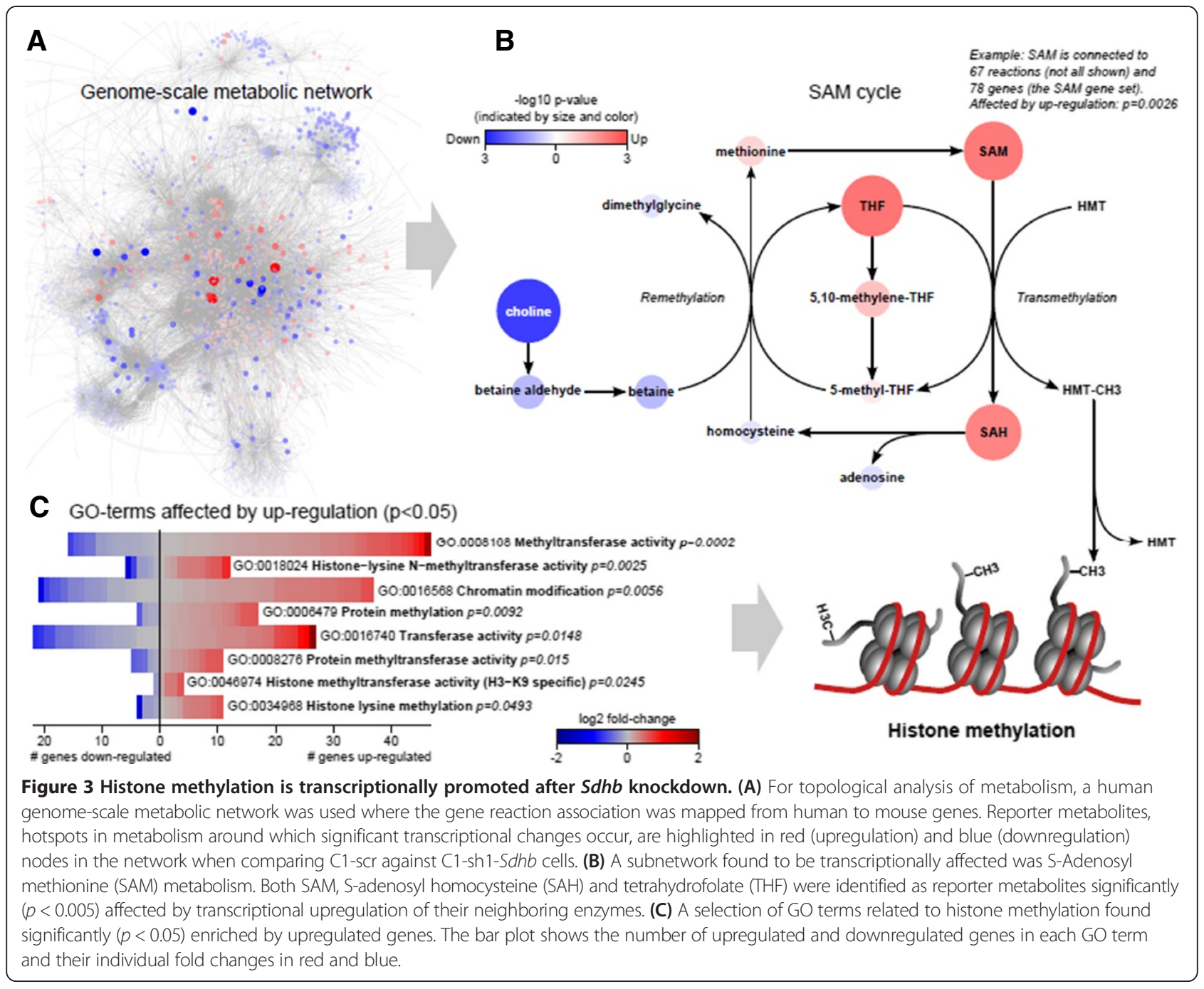

Twist2 mRNA relative to the DMSO-treated control cells as determined by qRT-PCR (Figure 4D). These data show that hypermethylation of $\mathrm{H} 3 \mathrm{~K} 27$ is sufficient to induce EMT, suggesting that the hypermethylated epigenome induced by the loss of $S d h b$ may be responsible for the EMT phenotype.

\section{Sdhb knockdown leads to reprogrammed glucose and glutamine utilization}

Given the fact that SDH is intricately involved in central carbon metabolism through its role in the TCA cycle and ETC, we examined global metabolite levels in the C1-scr and C1-sh1-Sdhb cells by LC-MS/MS. As expected, $S d h b$ knockdown cells had elevated levels of succinate and decreased levels of fumarate and malate relative to $\mathrm{C} 1$-scr cells (Figure 5A). This metabolic signature confirms the decrease in SDH activity upon $S d h b$ knockdown. In addition to altering TCA cycle intermediates succinate, fumarate, and malate, $S d h b$ knockdown affected many intracellular metabolites including amino acids, nucleotides, and intermediates in glycolysis as well as the pentose phosphate pathway (Additional file 7: Figure S4). Interestingly, $S d h b$ knockdown led to a significant increase of methylmalonic acid, possibly due to the feedback inhibition of succinate-CoA ligase (SUCL) by succinate (Additional file 7: Figure S4).

To further probe metabolic alterations caused by $S d h b$ knockdown, we used isotope-labeled nutrients combined with mass spectrometry-based techniques to trace glucose and glutamine through central carbon metabolism. Analysis of intracellular metabolites after culturing cells in the presence of uniformly labeled ${ }^{13} \mathrm{C}$-glucose ([U- $\left.{ }^{13} \mathrm{C}_{6}\right]$ glucose) revealed that $S d h b$ knockdown increased glucose contribution to sedoheptulose-7-phosphate and adenosine triphosphate (ATP); in contrast, glucose contribution to TCA cycle intermediate succinate was decreased (Figure 5B). Consistently, succinate and glutamate labeling from uniformly labeled ${ }^{13} \mathrm{C}$-glutamine $\left(\left[\mathrm{U}_{-}{ }^{13} \mathrm{C}_{5}\right]\right.$ glutamine) was increased (Figure 5C). This increased glutamine utilization by $S d h b$ knockdown cells is highlighted by their 


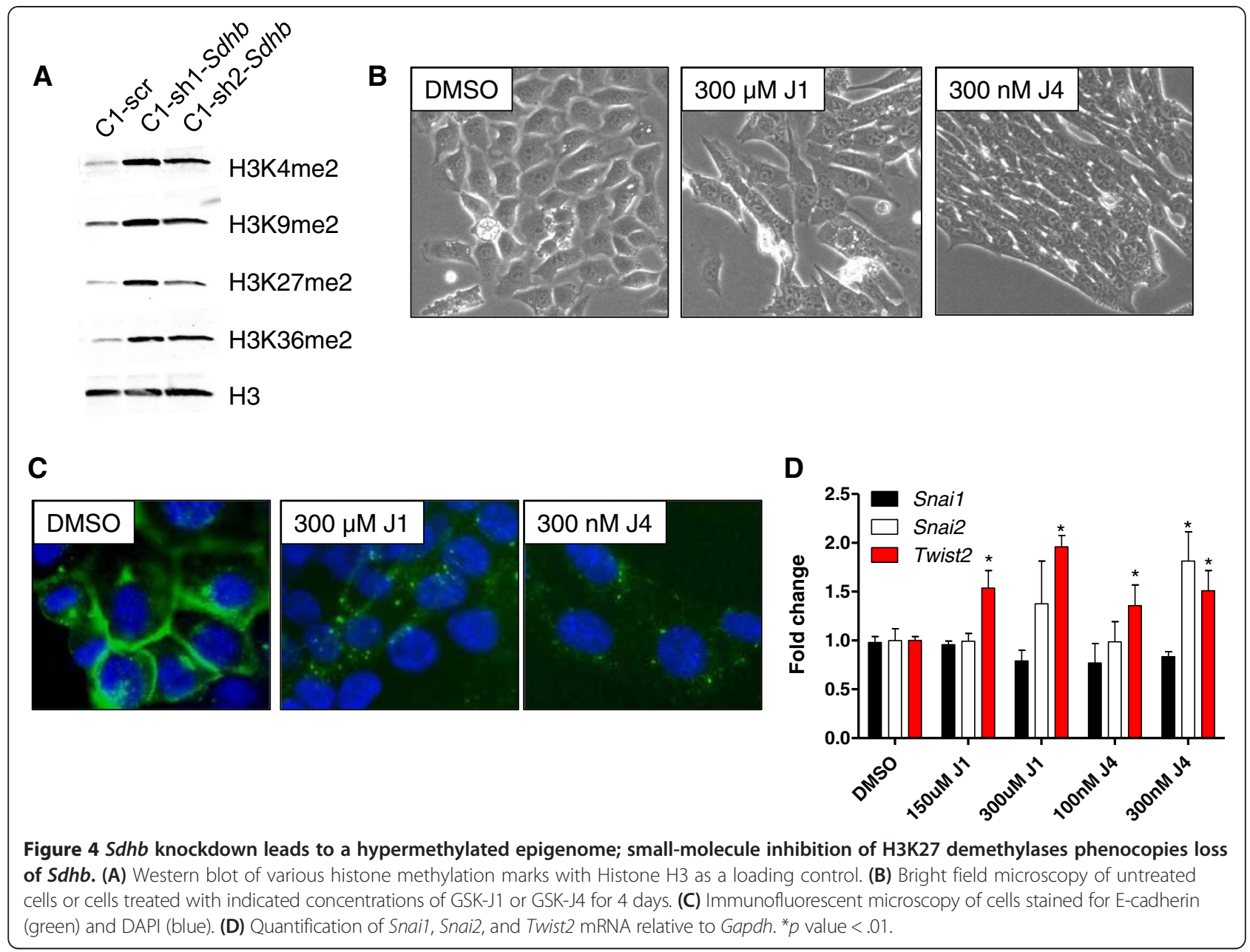

increased uptake of glutamine relative to the control cells (Figure 5D). These data suggest that $S d h b$ knockdown increases glucose contribution to the pentose phosphate pathway and nucleotide biosynthesis to support rapid proliferation. Increased nucleotide synthesis from glucose is achieved by decreasing glucose contribution to the TCA cycle, which is compensated by increasing glutamine contribution to the TCA cycle. Intracellular pool levels also support a model in which nucleotide biosynthesis is upregulated, as many glycolytic and pentose phosphate pathway intermediates are increased in response to $S d h b$ knockdown (Additional file 7: Figure S4).

Given the increased use of glutamine in $S d h b$ knockdown cells, we hypothesized that these cells have an augmented dependency on glutamine for survival relative to the control cells. To test this, we assayed the effect of varying glutamine concentration on cell proliferation. Indeed, in lower glutamine concentrations, C1-sh1-Sdhb cells grew slower in comparison to the control C1-scr cells (Figure 5E). Therefore, knockdown of $S d h b$ leads to a greater reliance on glutamine for cell survival.
Sdhb knockdown results in a mitochondrial reserve capacity defect that can be exploited by metformin Since $S d h b$ knockdown alters central carbon metabolism including the TCA cycle occurring in the mitochondria, we investigated the effect of $S d h b$ knockdown on mitochondrial activity. A key indicator of mitochondrial function is the oxygen consumption rate (OCR). To ascertain specific mitochondrial perturbations, the OCR of C1-scr and C1-sh1-Sdhb cells was determined during sequential treatment with compounds that modulate mitochondrial activity. Oligomycin, a $\mathrm{F}_{1} \mathrm{~F}_{0}$ ATP synthase inhibitor, blocks proton movement, thereby inhibiting oxidative phosphorylation (OXPHOS). FCCP reestablishes proton movement by dissipating the mitochondrial membrane potential to stimulate maximal $\mathrm{O}_{2}$ consumption and electron transport [43]. Finally, rotenone/myxothiazol, complex I and III inhibitors, completely block mitochondrial respiration. Both cell lines had similar basal OCR and responses to oligomycin and rotenone (Figure 6A). However, the cell lines responded differently to FCCP treatment (Figure 6A). The calculated difference between FCCP-induced and 


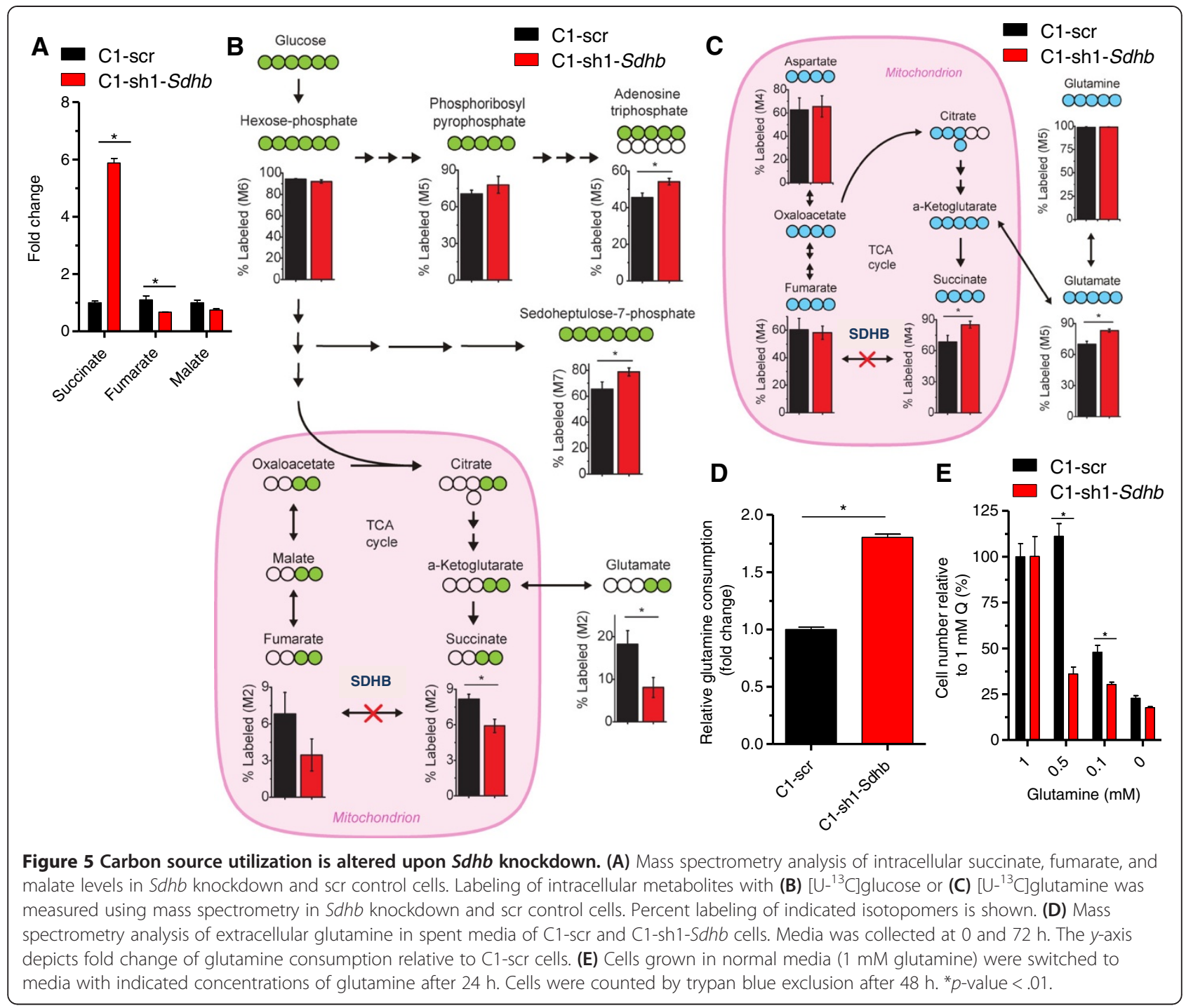

basal OCR indicates reserve respiratory capacity. FCCP injection increased respiration 59\% in C1-scr while spare respiratory capacity was nonexistent in C1-sh1-Sdhb cells (Figure 6A). Thus, C1-sh1-Sdhb cells respirate at their maximum capacity. $S D H B$ knockdown in HEY cells resulted in a lower basal respiration implying a greater reliance on $\mathrm{SDH}$ respiration in this particular cell line (Additional file 5: Figure S3C). Therefore, knockdown of $S d h b$ decreases mitochondrial reserve respiratory capacity.

The reserve respiratory capacity must be sufficient to provide enough ATP during times of energy stress. If ATP demands are not met, affected cells risk being driven into senescence or cell death [44]. To determine whether the decreased reserve capacity of Sdhb knockdown cells rendered them sensitive to energy stress, C1-scr and C1-sh1-Sdhb cells were cultured in normal media then switched to media with varying amounts of glucose. Despite the previous observation that $S d h b$ knockdown cells grew at a faster rate than control cells under normal growth conditions (Figure 2B), C1-sh1-Sdhb cells grew at a slower rate than $\mathrm{C} 1$-scr cells upon glucose withdrawal (Figure 6B). In fact, the majority of Sdhb knockdown cells appeared to undergo apoptosis (Figure 6C). Consistent with the increased dependence of C1-sh1-Sdhb cells on glucose relative to C1-scr cells, C1-sh1-Sdhb cells were more glycolytic (Figure 6D), took up glucose and excreted lactate at increased levels relative to $\mathrm{C} 1-\mathrm{scr}$ cells (Figure 6E). We suspect that the effect of glucose starvation on the Sdhb knockdown cells is twofold, a failure to adapt to the decrease in glycolytic ATP and its reliance on the pentose phosphate pathway for nucleotide biosynthesis.

Since C1-sh1-Sdhb cells are hypersensitive to glucose starvation, we were interested if this bioenergetics deficiency could be therapeutically exploited. Metformin, a commonly used antidiabetic drug, inhibits Complex I in 


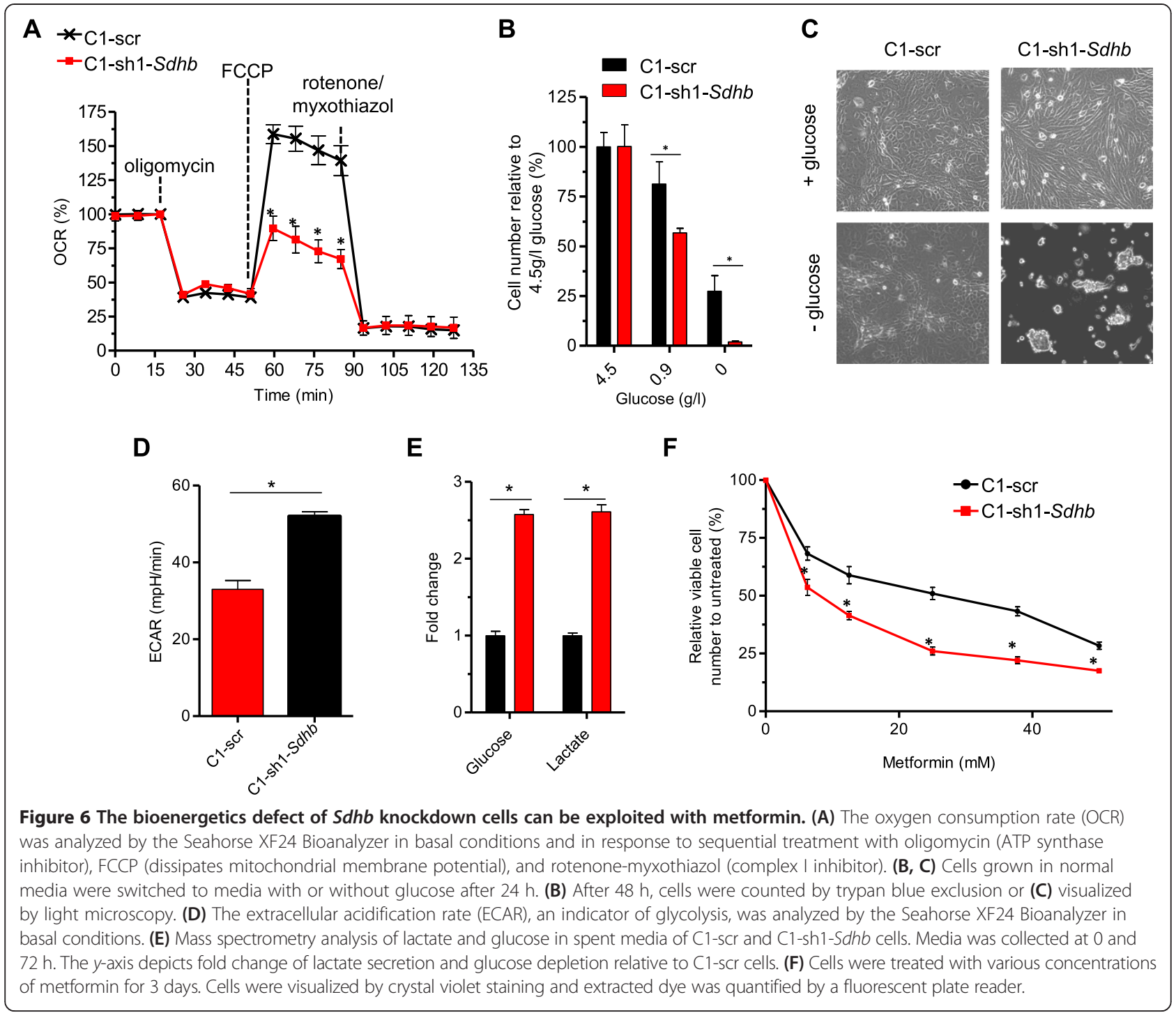

the ETC [45]. This reduces the cellular energy charge, a measure of the energetic state of the cell's adenine nucleotide pool defined as $([\mathrm{ATP}]+0.5[\mathrm{ADP}]) /([\mathrm{ATP}]+$ $[\mathrm{ADP}]+[\mathrm{AMP}])[46]$. To determine if metformin-induced energy stress had a differential effect on C1-scr and C1-sh1-Sdhb cells, cells were exposed to various concentrations of metformin. C1-sh1-Sdhb cells were hypersensitive to metformin treatment relative to $\mathrm{C} 1$-scr cells (Figure 6F). Specifically, metformin inhibited growth of the control and $S d h b$ knockdown cells with IC50 values of 29.73 and $14.73 \mathrm{mM}$, respectively (Figure 6F). Taken together, these results implicate a crucial role for Sdhb in the response to bioenergetic insult.

\section{Discussion}

Various mechanisms have been proposed for SDHassociated malignancy. Due to SDH's role in the ETC, it has been suggested that reactive oxygen species (ROS) can be induced by SDH-inactivating mutations, which contributes to genomic instability, DNA damage, and other tumorigenic phenomena [47]. However, Sdhb knockdown did not lead to altered ROS levels, and the antioxidant N-acetylcysteine (NAC) had no effect on various Sdhb knockdown phenotypes in our system (Additional file 8: Figure S5A). Interestingly, many studies have focused on the idea that succinate itself can act as an oncometabolite. Due to its structural similarity to $\alpha$-ketoglutarate $(\alpha-K G)$, succinate has been found to competitively inhibit $\alpha$-KG-dependent dioxygenases. This phenomenon was first described linking succinate to HIF1 $\alpha$ stabilization; succinate was found to directly inhibit prolyl hydroxylases which serve to degrade HIF1 $\alpha[48,49]$. However, we did not find altered HIF signaling between the control and $S d h b$ knockdown cells (Additional file 8: Figure S5B). This may be due in part to the modestly elevated levels of succinate in our $S d h b$ knockdown cells, 
approximately six-fold vs. control (Figure 5A), in comparison to previous studies (approximately 100-fold in tumors and approximately 300-fold upon complete knockout of Sdhb in mouse cells) $[16,50]$. It is also possible that HIF signaling by $S D H x$ mutations is tissue-dependent, thus explaining the specific range of cancers induced by $\mathrm{SDH}$ inactivation. Recently, the repertoire of $\alpha-\mathrm{KG}$ dependent dioxygenases targeted by succinate has expanded to include epigenome-modifying enzymes such as the TET family of 5-methylcytosine hydroxylases and histone demethylases [51]. Inhibition of these enzymes by elevated levels of succinate leads to genome-wide histone and DNA methylation [51,52]. In support of this idea, $S D H x$-related PGL tumors were found to display a hypermethylator phenotype, where epigenetic silencing was particularly severe in $S D H B$-mutated tumors [16]. This association between $S D H x$ mutations and hypermethylation was also found in GIST [53]. Our data further support this model in which SDH inhibition leads to a hypermethylated epigenome. It has been suggested that a stronger hypermethylator phenotype, which associates with stronger downregulation of target genes, may contribute to the metastatic phenotype [16]. Our results are in line with this idea as aberrant hypermethylation of H3K27 in mouse ovarian cancer cells was sufficient for EMT, thereby implicating the link between epigenetic remodeling and cell state [16]. However, overexpression of the H3K27 demethylases, UTX and JMJD3, in Sdhb knockdown cells failed to reverse EMT, thereby indicating a higher complexity to the maintenance of the mesenchymal state (Additional file 9: Figure S6). This is likely due to transcriptional upregulation of metabolic and epigenetic networks to support this hypermethylated state. Therefore, $\mathrm{SDH}$ dysfunction is a contributor of the tumorigenic process via mitochondrion-to-nuclear signaling.

In addition to SDH activity being linked to epigenetic reprogramming, we also found that $S d h b$ knockdown leads to reprogrammed glucose and glutamine utilization. Specifically, increased glucose flux into the pentose phosphate pathway and increased glutamine usage in the TCA cycle. Interestingly, these phenomena mimic disruption of another TCA cycle enzyme, fumarate hydratase (FH), in mouse kidney cells [54]. The mechanism by which SDH dysfunction leads to these effects is unknown. One possibility is that the global epigenetic changes induced by $S d h b$ knockdown may lead to an altered transcriptome as evidenced by pathway enrichment analysis of the RNAseq data (Additional file 7: Figure S4). The analysis indicated that the pentose phosphate pathway and nucleotide metabolism gene sets were both upregulated. The gene set analysis also showed that genes associated with oxidative phosphorylation were significantly downregulated (Additional file 10: Figure S7). In similar fashion, the $M Y C$ proto-oncogene has been shown to directly control the transcription of various genes involved in several metabolic pathways including glycolysis, the pentose phosphate pathway, and glutaminolysis [55,56]. Also, the glycolytic enzyme fructose-1,6-biphosphatase (FBP1) has been shown to influence metabolic reprogramming to impact EMT progression [57]. How SDH dysfunction affects the regulation of these enzymes will be an interesting subject of future research. Interestingly, an association between glutamine dependence and cancer invasiveness has been recently found in ovarian cancer, thereby providing further support for carbon source reprogramming to support different ovarian cancer states [58].

\section{Conclusions}

Although SDH dysfunction leads to enhanced cancer aggressiveness, it also results in metabolic vulnerabilities due to its involvement in both the TCA cycle and ETC. We showed that altered carbon source utilization in $S d h b$ knockdown cells renders them hypersensitive to both glucose and glutamine starvation. Also, by analyzing mitochondrial function, we found that SDH dysfunction leads to a decreased mitochondrial reserve capacity that can be exploited by energy stress caused by either glucose withdrawal or metformin treatment. Interestingly, it has been shown that combinatorial treatment with metformin and the glycolytic inhibitor 2-deoxyglucose are effective in a broad spectrum of pre-clinical cancer models [59]. Therefore, it is worth exploring the possibility that tumors with $S D H x$ mutations can be therapeutically targeted by exploiting their metabolic state.

\section{Additional files}

Additional file 1: Table S1. shRNA and GRT-PCR sequences.

Additional file 2: Supplementary materials and methods.

Additional file 3: Figure S1. SDHB protein expression in high-grade ovarian carcinoma. Representative images of tumors with (A) ubiquitous SDHB expression in epithelial and stromal cells and (B) decreased SDHB expression in epithelial cells relative to stromal cells. T, tumor; S, stroma.

Additional file 4: Figure S2. Immunofluorescence staining of SDHB in C1-scr and Sdhb knockdown cells.

Additional file 5: Figure S3. SDHB knockdown in HEY cells results in enhanced anchorage-independent growth and a defect in mitochondrial function. (A) Western blot detection of SDHB after shRNA-mediated knockdown. Complex $V$ alpha was used as a loading control. (B) Colony size in soft agar. (C) OCR was analyzed by the Seahorse XF24 Bioanalyzer in basal conditions and in response to sequential treatment with oligomycin, FCCP, and rotenone-myxothiazol. * $p$ value $<.01$.

Additional file 6: Table S2. Table of reporter metabolites and GO terms.

Additional file 7: Figure S4. Global analysis of metabolites in C1-scr and C1-sh1-Sdhb cells. Steady state levels of metabolites in control and Sdhb knockdown cells were determined by mass spectrometry. A heat map was generated using Cluster 3.0 for unsupervised clustering and JavaTreeView for visualization.

Additional file 8: Figure S5. Sdhb knockdown does not affect ROS levels or HIF signaling in C1 cells. (A) C1-scr and C1-sh1-Sdhb cells were incubated with MitoTracker ${ }^{\circledR}$ Red $\mathrm{CM}-\mathrm{H} 2 \mathrm{X}$ Ros to determine relative ROS 
levels. No significant difference in probe intensity was observed. (B) HIF-response element luciferase activity in Sdhb knockdown and control cells. Data are normalized to renilla luciferase activity and shown relative to $\mathrm{C1}$-scr cells.

Additional file 9: Figure S6. Overexpression of H3K27 demethylases, JMJD3 and UTX, does not promote MET in Sdhb knockdown cells. C1-sh1-Sdhb and C1-sh2-Sdhb cells were transfected with HA-tagged JMJD3, UTX, or GFP constructs. Lysates were collected 7 days post-transfection. Western blot detection of HA-tagged JMJD3 and UTX, E-cadherin, and $\beta$-actin.

Additional file 10: Figure S7. Sdhb knockdown affects the transcriptional regulation of the metabolism of amino acids, nucleotides, and oxidative phosphorylation. In order to identify metabolic pathways influenced by transcriptional regulation, we performed a gene set analysis using the pathways defined by the human genome-scale metabolic network HMR2. The resulting heat map shows the top significant pathways affected by transcriptional changes. The leftmost and rightmost columns show $p$ values for the pathways being coordinately regulated in a distinct direction. The second columns from the left and right show the $p$ values for pathways having a significant subset of upregulated or downregulated genes. The middle column shows the $p$ values for pathways affected by transcriptional regulation in general, regardless of the direction. The pentose phosphate pathway, providing backbones for both nucleotides and amino acids, is upregulated. This pattern is also seen for pathways related to nucleotide metabolism and amino acid metabolism (marked in red). Oxidative phosphorylation is significantly downregulated (marked in blue). For the relevant pathways, $p$ values $<0.04$ are marked with a black box.

\section{Abbreviations}

SDH: succinate dehydrogenase; EMT: epithelial-to-mesenchymal transition; TCA: the citric acid cycle; ETC.: electron transport chain; SDHAF1/ SDHAF2: succinate dehydrogenase assembly factor; PCC: hereditary pheochromocytoma; PGL: paraganglioma; GIST: gastrointestinal stromal tumor; FCCP: carbonyl cyanide 4-(trifluoromethoxy)phenylhydrazone; TCGA: the Cancer Genome Atlas; CNA: copy number alterations; HMR: human genome-scale metabolic network; SAM: S-adenosyl methionine; SAH: S-adenosyl homocysteine; THF: tetrahydrofolate; OCR: oxygen consumption rate; OXPHOS: oxidative phosphorylation; ECAR: extracellular acidification rate; ROS: reactive oxygen species; NAC: N-acetylcysteine; FH: fumarate hydratase.

\section{Competing interests}

The authors declare that they have no competing interests.

\section{Authors' contributions}

PJA designed all experiments, interpreted results, and wrote the manuscript. SO participated in the design of all experiments and the writing of the manuscript. SYL performed all mass spectrometry experiments and their interpretation and helped write the manuscript. LV performed the Seahorse Bioanalyzer experiments. KR helped with interpretation of the Seahorse experiments. LV performed and interpreted the metabolic gene network analysis and helped write the manuscript. JN assisted with interpretation of the metabolic gene network analysis and helped write the manuscript. MG helped with RT-PCR. JAB assisted with Western blotting analysis. BS assisted with proliferation assays. WRW helped interpret results and provided feedback on the project. BYK helped interpret results and provided feedback on the project. All authors read and approved the final manuscript.

\section{Acknowledgements}

This work was supported by the Foundation for Women's Cancer Lynette Medlin-Brumfield/Mary-Jane Welker Ovarian Cancer Early Detection Research Grant (P-J.A.). Work in the lab of S.O. is supported, in part, by grants from Alex's Lemonade Stand Foundation, Margaret E. Early Medical Research Trust, Sandy Rollman Foundation, Department of Defense Ovarian Cancer Clinical Translational Leverage Award (W81XWH-13-OCRP-TLA) and the American Cancer Society (RSG-10-252-01-TBG). Work in the lab of K.R. is supported, in part, by the National Institutes of Health grants P01 HL28481 and NCRR S10RR026744. W.R.W. is supported, in part, by the Ovarian Cancer Research Fund Liz Tilberis Scholarship. S.Y.L. is supported by the Department of
Defense CDMRP Visionary Postdoctoral Award under award number W81XWH-12-1-0466. Views and opinions of, and endorsements by, the authors do not reflect those of the US Army or the Department of Defense. The authors thank the MSU Mass Spectrometry Core and Gavin Reid and Eran Andrechek for access to their laboratories. We would also like to thank Kristian Helin for the UTX and JMJD3 constructs.

\section{Author details}

${ }^{1}$ Women's Cancer Program, Samuel Oschin Comprehensive Cancer Institute, Cedars-Sinai Medical Center, 8700 Beverly Blvd., Los Angeles, CA 90048, USA. ${ }^{2}$ Department of Physiology, Michigan State University, East Lansing, MI 48824, USA. ${ }^{3}$ Koch Institute for Integrative Cancer Research, Massachusetts Institute of Technology, Cambridge, MA 02139, USA. ${ }^{4}$ Systems and Synthetic Biology, Department of Chemical and Biological Engineering, Chalmers University of Technology, Gothenburg 41296, Sweden. ${ }^{5}$ Department of Human Genetics, David Geffen School of Medicine, University of California Los Angeles, Los Angeles, CA 90095, USA. ${ }^{6}$ Graduate Program in Biomedical Science and Translational Medicine, Cedars-Sinai Medical Center, Los Angeles, CA 90048, USA. ${ }^{7}$ Department of Obstetrics and Gynecology, David Geffen School of Medicine, University of California Los Angeles, Los Angeles, CA 90095, USA.

Received: 20 May 2014 Accepted: 4 September 2014

Published: 15 December 2014

\section{References}

1. Siegel R, Naishadham D, Jemal A: Cancer statistics, 2013. CA Cancer J Clin 2013, 63:11-30.

2. SEER Cancer Statistics Review, 1975-2010. [http://seer.cancer.gov/csr/ 1975_2010/]

3. Seidman JD, Horkayne-Szakaly I, Haiba M, Boice CR, Kurman RJ, Ronnett BM: The histologic type and stage distribution of ovarian carcinomas of surface epithelial origin. Int J Gynecol Pathol 2004, 23:41-44.

4. Cancer Genome Atlas Research N: Integrated genomic analyses of ovarian carcinoma. Nature 2011, 474:609-615.

5. Zhang W, Liu Y, Sun N, Wang D, Boyd-Kirkup J, Dou X, Han JD: Integrating genomic, epigenomic, and transcriptomic features reveals modular signatures underlying poor prognosis in ovarian cancer. Cell Rep 2013, 4:542-553.

6. De Craene B, BerX G: Regulatory networks defining EMT during cancer initiation and progression. Nat Rev Cancer 2013, 13:97-110.

7. Nieto MA: The ins and outs of the epithelial to mesenchymal transition in health and disease. Annu Rev Cell Dev Biol 2011, 27:347-376.

8. Warburg O: Origin of cancer cells. Oncologia 1956, 9:75-83.

9. Lunt SY, Vander Heiden MG: Aerobic glycolysis: meeting the metabolic requirements of cell proliferation. Annu Rev Cell Dev Biol 2011, 27:441-464.

10. Gatto F, Nookaew I, Nielsen J: Chromosome 3p loss of heterozygosity is associated with a unique metabolic network in clear cell renal carcinoma. Proc Natl Acad Sci U S A 2014, 111:E866-E875.

11. Bardella C, Pollard PJ, Tomlinson I: SDH mutations in cancer. Biochim Biophys Acta 2011, 1807:1432-1443.

12. King A, Selak MA, Gottlieb E: Succinate dehydrogenase and fumarate hydratase: linking mitochondrial dysfunction and cancer. Oncogene 2006, 25:4675-4682.

13. Hoekstra AS, Bayley JP: The role of complex II in disease. Biochim Biophys Acta 2013, 1827:543-551.

14. Finley LW, Haas W, Desquiret-Dumas V, Wallace DC, Procaccio V, Gygi SP, Haigis MC: Succinate dehydrogenase is a direct target of sirtuin 3 deacetylase activity. PLoS One 2011, 6:e23295.

15. Loriot C, Burnichon N, Gadessaud N, Vescovo L, Amar L, Libe R, Bertherat J, Plouin PF, Jeunemaitre X, Gimenez-Roqueplo AP, Favier J: Epithelial to mesenchymal transition is activated in metastatic pheochromocytomas and paragangliomas caused by SDHB gene mutations. J Clin Endocrinol Metab 2012, 97:E954-E962.

16. Letouze E, Martinelli C, Loriot C, Burnichon N, Abermil N, Ottolenghi C, Janin M, Menara M, Nguyen AT, Benit P, Buffet A, Marcaillou C, Bertherat J, Amar $L$, Rustin P, De Reyniès $A$, Gimenez-Roqueplo AP, Favier J: SDH mutations establish a hypermethylator phenotype in paraganglioma. Cancer Cell 2013, 23:739-752.

17. Liu J, Gao L, Zhang H, Wang D, Wang M, Zhu J, Pang C, Wang C: Succinate dehydrogenase $5(\mathrm{SDH} 5)$ regulate (GSK)-3beta-beta-catenin-mediated lung cancer metastasis. J Biol Chem 2013, 288:29965-29973. 
18. Zhang T, Wu X, Ke C, Yin M, Li Z, Fan L, Zhang W, Zhang H, Zhao F, Zhou X, Lou G, Li K: Identification of potential biomarkers for ovarian cancer by urinary metabolomic profiling. J Proteome Res 2013, 12:505-512.

19. Miao J, Wang Z, Provencher H, Muir B, Dahiya S, Carney E, Leong CO, Sgroi DC, Orsulic S: HOXB13 promotes ovarian cancer progression. Proc Natl Acad Sci U S A 2007, 104:17093-17098.

20. Orsulic S: An RCAS-TVA-based approach to designer mouse models. Mamm Genome 2002, 13:543-547.

21. Xing D, Orsulic S: A genetically defined mouse ovarian carcinoma model for the molecular characterization of pathway-targeted therapy and tumor resistance. Proc Natl Acad Sci U S A 2005, 102:6936-6941.

22. Cerami E, Gao J, Dogrusoz U, Gross BE, Sumer SO, Aksoy BA, Jacobsen A, Byrne CJ, Heuer ML, Larsson E, Antipin Y, Reva B, Goldberg AP, Sander C, Schultz N: The cBio cancer genomics portal: an open platform for exploring multidimensional cancer genomics data. Cancer Discov 2012, 2:401-404.

23. Beroukhim R, Mermel CH, Porter D, Wei G, Raychaudhuri S, Donovan J, Barretina J, Boehm JS, Dobson J, Urashima M, Mc Henry KT, Pinchback RM, Ligon AH, Cho YJ, Haery L, Greulich H, Reich M, Winckler W, Lawrence MS, Weir BA, Tanaka KE, Chiang DY, Bass AJ, Loo A, Hoffman C, Prensner J, Liefeld T, Gao Q, Yecies D, Signoretti S, et al: The landscape of somatic copy-number alteration across human cancers. Nature 2010, 463:899-905.

24. Munger J, Bennett BD, Parikh A, Feng XJ, McArdle J, Rabitz HA, Shenk T, Rabinowitz JD: Systems-level metabolic flux profiling identifies fatty acid synthesis as a target for antiviral therapy. Nat Biotechnol 2008, 26:1179-1186.

25. Clasquin MF, Melamud E, Rabinowitz JD: LC-MS data processing with MAVEN: a metabolomic analysis and visualization engine. Curr Protoc Bioinformatics 2012, Chapter 14:Unit14 11

26. Melamud E, Vastag L, Rabinowitz JD: Metabolomic analysis and visualization engine for LC-MS data. Anal Chem 2010, 82:9818-9826.

27. Millard P, Letisse F, Sokol S, Portais JC: IsoCor: correcting MS data in isotope labeling experiments. Bioinformatics 2012, 28:1294-1296.

28. Plaisier CL, Bennett BJ, He A, Guan B, Lusis AJ, Reue K, Vergnes L: Zbtb16 has a role in brown adipocyte bioenergetics. Nutr Diabetes 2012, 2:e46.

29. Trapnell C, Roberts A, Goff L, Pertea G, Kim D, Kelley DR, Pimentel H, Salzberg SL, Rinn JL, Pachter L: Differential gene and transcript expression analysis of RNA-seq experiments with TopHat and Cufflinks. Nat Protoc 2012, 7:562-578.

30. Varemo L, Nielsen J, Nookaew I: Enriching the gene set analysis of genome-wide data by incorporating directionality of gene expression and combining statistical hypotheses and methods. Nucleic Acids Res 2013, 41:4378-4391.

31. Mardinoglu A, Agren R, Kampf C, Asplund A, Uhlen M, Nielsen J: Genomescale metabolic modeling of hepatocytes leads to identification of serine deficiency in non-alcoholic fatty liver disease. Nat Comm 2013, 5:3083.

32. Durinck S, Moreau Y, Kasprzyk A, Davis S, De Moor B, Brazma A, Huber W: BioMart and Bioconductor: a powerful link between biological databases and microarray data analysis. Bioinformatics 2005, 21:3439-3440.

33. Solimini NL, Xu Q, Mermel CH, Liang AC, Schlabach MR, Luo J, Burrows AE, Anselmo AN, Bredemeyer AL, Li MZ, Beroukhim R, Meyerson M, Elledge SJ: Recurrent hemizygous deletions in cancers may optimize proliferative potential. Science 2012, 337:104-109.

34. Davoli T, XU AW, Mengwasser KE, Sack LM, Yoon JC, Park PJ, Elledge SJ: Cumulative haploinsufficiency and triplosensitivity drive aneuploidy patterns and shape the cancer genome. Cell 2013, 155:948-962.

35. Tam WL, Weinberg RA: The epigenetics of epithelial-mesenchymal plasticity in cancer. Nat Med 2013, 19:1438-1449.

36. Shiota M, Izumi H, Onitsuka T, Miyamoto N, Kashiwagi E, Kidani A, Yokomizo A, Naito S, Kohno K: Twist promotes tumor cell growth through YB-1 expression. Cancer Res 2008, 68:98-105.

37. Ansieau S, Bastid J, Doreau A, Morel AP, Bouchet BP, Thomas C, Fauvet F, Puisieux I, Doglioni C, Piccinin S, Maestro R, Voeltzel T, Selmi A, ValsesiaWittmann S, Caron de Fromentel C, Puisieux A: Induction of EMT by twist proteins as a collateral effect of tumor-promoting inactivation of premature senescence. Cancer Cell 2008, 14:79-89.

38. Qian J, Luo Y, Gu X, Zhan W, Wang X: Twist1 promotes gastric cancer cell proliferation through up-regulation of FoxM1. PLoS One 2013, 8:e77625.

39. Mardinoglu A, Agren R, Kampf C, Asplund A, Uhlen M, Nielsen J: Genome-scale metabolic modelling of hepatocytes reveals serine deficiency in patients with non-alcoholic fatty liver disease. Nat Commun 2014, 5:3083.
40. Patil KR, Nielsen J: Uncovering transcriptional regulation of metabolism by using metabolic network topology. Proc Natl Acad Sci U S A 2005, 102:2685-2689.

41. Martinez-Garcia E, Licht JD: Deregulation of H3K27 methylation in cancer. Nat Genet 2010, 42:100-101.

42. Kruidenier L, Chung CW, Cheng Z, Liddle J, Che K, Joberty G, Bantscheff M, Bountra C, Bridges A, Diallo H, Eberhard D, Hutchinson S, Jones E, Katso R, Leveridge M, Mander PK, Mosley J, Ramirez-Molina C, Rowland P, Schofield CJ, Sheppard RJ, Smith JE, Swales C, Tanner R, Thomas P, Tumber A, Drewes G, Oppermann U, Patel DJ, Lee K, et al: A selective jumonji H3K27 demethylase inhibitor modulates the proinflammatory macrophage response. Nature 2012, 488:404-408.

43. Heytler PG: Uncouplers of oxidative phosphorylation. Methods Enzymol 1979, 55:462-442.

44. Desler C, Hansen TL, Frederiksen JB, Marcker ML, Singh KK, Juel Rasmussen $\mathrm{L}$ : Is there a link between mitochondrial reserve respiratory capacity and aging? J Aging Res 2012, 2012:192503.

45. El-Mir MY, Nogueira V, Fontaine E, Averet N, Rigoulet M, Leverve X: Dimethylbiguanide inhibits cell respiration via an indirect effect targeted on the respiratory chain complex I. J Biol Chem 2000, 275:223-228.

46. Miller RA, Birnbaum MJ: An energetic tale of AMPK-independent effects of metformin. J Clin Invest 2010, 120:2267-2270.

47. Kaelin WG Jr: SDH5 mutations and familial paraganglioma: somewhere Warburg is smiling. Cancer Cell 2009, 16:180-182.

48. Pollard PJ, Briere JJ, Alam NA, Barwell J, Barclay E, Wortham NC, Hunt T, Mitchell M, Olpin S, Moat SJ, Hargreaves IP, Heales SJ, Chung YL, Griffiths JR, Dalgleish A, McGrath JA, Gleeson MJ, Hodgson SV, Poulsom R, Rustin P, Tomlinson IP: Accumulation of Krebs cycle intermediates and overexpression of HIF1alpha in tumours which result from germline $\mathrm{FH}$ and SDH mutations. Hum Mol Genet 2005, 14:2231-2239.

49. Selak MA, Armour SM, MacKenzie ED, Boulahbel H, Watson DG, Mansfield KD, Pan Y, Simon MC, Thompson CB, Gottlieb E: Succinate links TCA cycle dysfunction to oncogenesis by inhibiting HIF-alpha prolyl hydroxylase. Cancer Cell 2005, 7:77-85.

50. Imperiale A, Moussallieh FM, Sebag F, Brunaud L, Barlier A, Elbayed K, Bachellier P, Goichot B, Pacak K, Namer IJ, Taieb D: A new specific succinate-glutamate metabolomic hallmark in SDHx-related paragangliomas. PLoS One 2013, 8:e80539.

51. Xiao M, Yang H, Xu W, Ma S, Lin H, Zhu H, Liu L, Liu Y, Yang C, Xu Y, Zhao $S$, Ye D, Xiong Y, Guan KL: Inhibition of alpha-KG-dependent histone and DNA demethylases by fumarate and succinate that are accumulated in mutations of FH and SDH tumor suppressors. Genes Dev 2012, 26:1326-1338.

52. Cervera AM, Bayley JP, Devilee P, McCreath KJ: Inhibition of succinate dehydrogenase dysregulates histone modification in mammalian cells. Mol Cancer 2009, 8:89.

53. Killian JK, Kim SY, Miettinen M, Smith C, Merino M, Tsokos M, Quezado M, Smith WI Jr, Jahromi MS, Xekouki P, Szarek E, Walker RL, Lasota J, Raffeld M, Klotzle B, Wang Z, Jones L, Zhu Y, Wang Y, Waterfall JJ, O'Sullivan MJ, Bibikova M, Pacak K, Stratakis C, Janeway KA, Schiffman JD, Fan JB, Helman L, Meltzer PS: Succinate dehydrogenase mutation underlies global epigenomic divergence in gastrointestinal stromal tumor. Cancer Discov 2013, 3:648-657.

54. Frezza C, Zheng L, Folger O, Rajagopalan KN, Mackenzie ED, Jerby L, Micaroni M, Chaneton B, Adam J, Hedley A, Kalna G, Tomlinson IP, Pollard PJ, Watson DG, Deberardinis RJ, Shlomi T, Ruppin E, Gottlieb E: Haem oxygenase is synthetically lethal with the tumour suppressor fumarate hydratase. Nature 2011, 477:225-228.

55. Dang CV: MYC, metabolism, cell growth, and tumorigenesis. Cold Spring Harb Perspect Med 2013, 3:a014217.

56. Liu W, Le A, Hancock C, Lane AN, Dang CV, Fan TW, Phang JM: Reprogramming of proline and glutamine metabolism contributes to the proliferative and metabolic responses regulated by oncogenic transcription factor c-MYC. Proc Natl Acad Sci U S A 2012, 109:8983-8988.

57. Dong C, Yuan T, Wu Y, Wang Y, Fan TW, Miriyala S, Lin Y, Yao J, Shi J, Kang T, Lorkiewicz P, St Clair D, Hung MC, Evers BM, Zhou BP: Loss of FBP1 by snail-mediated repression provides metabolic advantages in basal-like breast cancer. Cancer Cell 2013, 23:316-331.

58. Yang L, Moss T, Mangala LS, Marini J, Zhao H, Wahlig S, Armaiz-Pena G, Jiang D, Achreja A, Win J, Roopaimoole R, Rodriguez-Aguayo C, MercadoUribe I, Lopez-Berestein G, Liu J, Tsukamoto T, Sood AK, Ram PT, Nagrath D: 
Metabolic shifts toward glutamine regulate tumor growth, invasion and bioenergetics in ovarian cancer. Mol Syst Biol 2014, 10:728.

59. Cheong JH, Park ES, Liang J, Dennison JB, Tsavachidou D, Nguyen-Charles C, Wa Cheng K, Hall H, Zhang D, Lu Y, Ravoori M, Kundra V, Ajani J, Lee JS,

Ki Hong W, Mills GB: Dual inhibition of tumor energy pathway by

2-deoxyglucose and metformin is effective against a broad spectrum of preclinical cancer models. Mol Cancer Ther 2011, 10:2350-2362.

doi:10.1186/2049-3002-2-21

Cite this article as: Aspuria et al:: Succinate dehydrogenase inhibition

leads to epithelial-mesenchymal transition and reprogrammed carbon metabolism. Cancer \& Metabolism 2014 2:21

\section{Submit your next manuscript to BioMed Central and take full advantage of:}

- Convenient online submission

- Thorough peer review

- No space constraints or color figure charges

- Immediate publication on acceptance

- Inclusion in PubMed, CAS, Scopus and Google Scholar

- Research which is freely available for redistribution 